\title{
Crystallographic excursion in superspace
}

\author{
Gervais Chapuis* \\ Laboratory of crystallography, Swiss Federal School of Technology (EPFL), BSP, \\ 1015 Lausanne, Switzerland
}

Received 22 September 2003; accepted 10 April 2004

\begin{abstract}
After studying more than 100 different samples of calaverite $\operatorname{Au}_{1-p} \operatorname{Ag}_{p} \mathrm{Te}_{2}(p<0.15)$ three famous mineralogists declared in a well documented 1931 publication the invalidity of the law of rational indices. What could lead them to draw such an extreme conclusion from the observation law of Hauÿ dating from more than two centuries? Their observations remained unexplained for the next forty years. In the 1970's, the room temperature structure of $\gamma-\mathrm{Na}_{2} \mathrm{CO}_{3}$ resisted any attempt for a precise structural analysis. The appearance of satellite reflections was noted on single crystal diffractograms which led to the generalisation of the concept of crystal. This generalisation consisted in using at least four integers to fully characterise each individual diffractions peaks. The theory of periodic crystals in space of higher dimension, i.e. the superspace was then developed to deal with the new experimental observations. Later, a new class of materials called composite crystals and still later, the discovery of quasicrystals only reinforced the validity of the superspace concept to describe any material requiring more than three integers to index their diffraction pattern.

What is the essence of superspace to describe crystalline structures? Any crystal structure requesting more than three integers to index its diffraction pattern can be described as a periodic object in superspace with dimension equal to the number of required integer. The structure observed in our real word is a three dimensional cut of the superspace description. In general this cut is irrational and consequently the crystal is aperiodic. Calaverite and $\gamma-$ $\mathrm{Na}_{2} \mathrm{CO}_{3}$ are examples of aperiodic crystals which includes incommensurately modulated crystals, composite and quasi-crystals. Rational cuts are also possible. In this case, the structure is periodic and is usually called a superstructure.
\end{abstract}

(C) 2004 Elsevier Ltd. All rights reserved.

\footnotetext{
${ }^{*}$ Tel./fax: +41 21693 0630/0634.

E-mail address: gervais.chapuis@epfl.ch (G. Chapuis).
} 
Keywords: Commensurately modulated structures; Incommensurately modulated structures; Aperiodic crystal structures; Superstructures; Superspace

\section{Introduction}

In 1931, three authors, Goldschmidt, Palache and Peacock (GPP), after optically studying more than 100 samples of the mineral calaverite $\mathrm{Au}_{1-p} \mathrm{Ag}_{p} \mathrm{Te}_{2}(p<0.15)$, claimed that the law of rational indices is not of general validity [1]. The law of rational indices has been known from the work of Haüy since 1784 and expresses the property that each crystalline face can be uniquely and unambiguously characterized by three, in general, small integers. The three authors had to present serious arguments in order to question one of the most fundamental pillars of mineralogy. Indeed, their article, covering 58 pages, not including many bicolour tables, is an example of a very fine and well documented scientific study put forward in order to substantiate their claim. This claim was probably not generally accepted by the scientific community and the paper remained nearly forgotten on the library shelves.

Surprisingly, the method of X-ray diffraction, which was developed by the Braggs a quarter of a century before, was not immediately applied at least to find a clue for the failure of the law of rational indices for calaverite. The close relationship between the lattice planes which give rise to diffraction in the Braggs interpretation and the faces which are characteristic for each natural or synthetic crystalline sample is based on the lattice periodicity of the structure of matter. This lattice periodicity is defined by three basis vectors $\mathbf{a}_{i}$ which forms a parallelepiped, the so-called unit cell, which repeats itself to infinity in the three dimensions of space. Directly associated to the basis vectors, three corresponding reciprocal vectors satisfying the conditions $\mathbf{a}_{i}^{*} \cdot \mathbf{a}_{j}=\delta_{i j}$ ( 1 if $i=j$ and 0 if $i \neq j$ ) are also useful. The interesting property of reciprocal vectors is that each diffracted intensity $\mathrm{I}(\mathbf{h})$ can be associated with a reciprocal vector $\mathbf{h}=h_{1} \mathbf{a}_{1}^{*}+h_{2} \mathbf{a}_{2}^{*}+h_{3} \mathbf{a}_{3}^{*}$ with integers $h_{i}$, and each crystalline face can also be described by its normal, the same vector $\mathbf{h}$ with integer coefficients. These three coefficients are known as Miller indices. This property to index each face with three integers was indeed questioned by the authors following their study on the mineral calaverite.

If the law of rational indices is not satisfied for calaverite, the corresponding $\mathrm{X}$-ray diffraction pattern should exhibit some anomaly supporting the affirmation of the authors. In other words, diffractograms of calaverite should show at least some reflections which cannot be indexed by integer multiples of the reciprocal basis vectors. This attempt occurred four years later, when Tunell and Ksanda [2] attempted to link the "adventive diffraction spots" they observed on diffractograms with the orientation of the faces of calverite. Unfortunately, this attempt failed partly.

The next major step in attempting to understand the implication of the GPP paper occurred in the 1960s, when Brouns, Visser and de Wolff found anomalies in the diffractograms of $\mathrm{Na}_{2} \mathrm{CO}_{3}$ [3]. After facing some difficulties in indexing a powder diagram of this substance, they managed to obtain a diffraction pattern from a 
single crystal sample. The complete indexation of the diagram was only possible by postulating an additional reciprocal dimension independent of the three usual ones. All the reflections could be expressed in the form $\mathbf{H}=h_{1} \mathbf{a}_{1}^{*}+h_{2} \mathbf{a}_{2}^{*}+h_{3} \mathbf{a}_{3}^{*}+m \mathbf{q}$. Reflections with $m=0$ were called main reflections, whereas for $m \neq 0$ they were called satellite reflections (see adventive diffraction spots above). It was soon realised that the difficulties in indexing the faces of calaverite and the existence of satellite reflections were two aspects of the same phenomenon. However, it took another decade for de Wolff to develop the necessary tools to analyse the so-called modulated structure [4]. In the following section, we shall present the concept of superspace, which has been introduced in order to study the types of crystalline structures mentioned above, which do not conform to the classical description which is still valid for a large number of cases. This concept is now well established and has been successfully applied to a large number of aperiodic crystal structures.

\section{The concept of superspace}

One of the common features observed in calaverite and $\mathrm{Na}_{2} \mathrm{CO}_{3}$ is that their diffraction pattern can be indexed with four integer indices, which are the coefficients of vector $\mathbf{H}$ described above. Currently, many examples are known where up to six integer coefficients (e.g. icosahedral quasicrystals) must be introduced in order to fully describe the diffraction pattern. In this context and without losing generality, we shall limit ourselves to four components. In his study of sodium carbonate, de Wolff had the brilliant idea of interpreting the diffractogram in a space of higher dimension, four in this particular case. Fig. 1 illustrates the embedding of the dif-

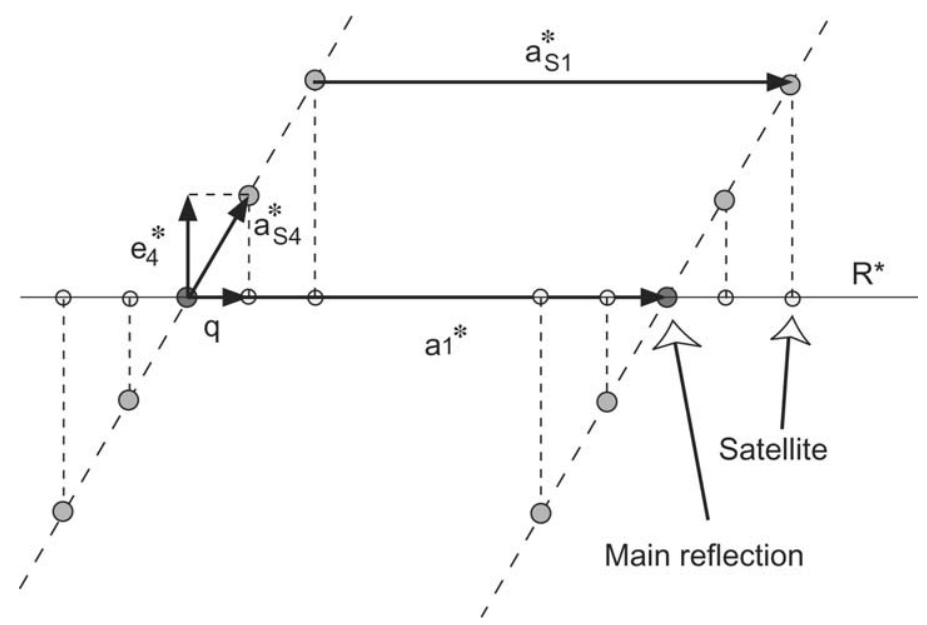

Fig. 1. Four-dimensional extension of the reciprocal space. Index $\mathrm{s}$ refer to superspace. $\mathbf{a}_{\mathrm{S} 4}$ is decomposed into two components, $\mathbf{e}_{4}^{*}$ normal to $R^{*}$, the $3 \mathrm{~d}$ reciprocal space and $\mathbf{q}$, the components of the satellite in $R^{*}$. 
fraction pattern in $4 \mathrm{~d}$ space. In this model, the satellite reflections which are observed experimentally are decomposed into two components, one normal and one parallel to the $3 \mathrm{~d}$-reciprocal space.

We have thus seen that the number of integer indices necessary to index a diffraction pattern (the rank) is equal to or higher than the dimension of space in which the crystal is described. If the rank and the dimension are equal, the crystal is called periodic, whereas if the rank is higher, the crystal is called aperiodic. Incommensurate, composite and quasicrystals all belong to the category of aperiodic crystals.

At this point, the reader has probably realised that the definitions presented here deal with reciprocal space only. In order to describe the structure of aperiodic crystals in real space, we shall introduce here the notion of superspace. The dimension of superspace in which a structure is described is equal to its rank. Fig. 2 illustrates the physical concept which is used to describe an aperiodic structure. In this particular case, each atom is represented by a modulation curve (one dimensional curve) which is periodically repeated along the four dimensions of superspace. The corresponding crystal structure observed in the real world is a $3 \mathrm{~d}$ intersection of this periodic object in a higher dimension. This section is, however, not periodic if at least one coefficient of the modulation vector $\mathbf{q}=\alpha \mathbf{a}_{1}^{*}+\beta \mathbf{a}_{2}^{*}+\gamma \mathbf{a}_{3}^{*}$ is irrational. On the line indicated by $R$, which represents the three dimensional space, atoms $\mathrm{A}$, $\mathrm{B}$ and $\mathrm{C}$ are not equally spaced $(\mathrm{AB} \neq \mathrm{BC})$. The resulting structure is aperiodic.

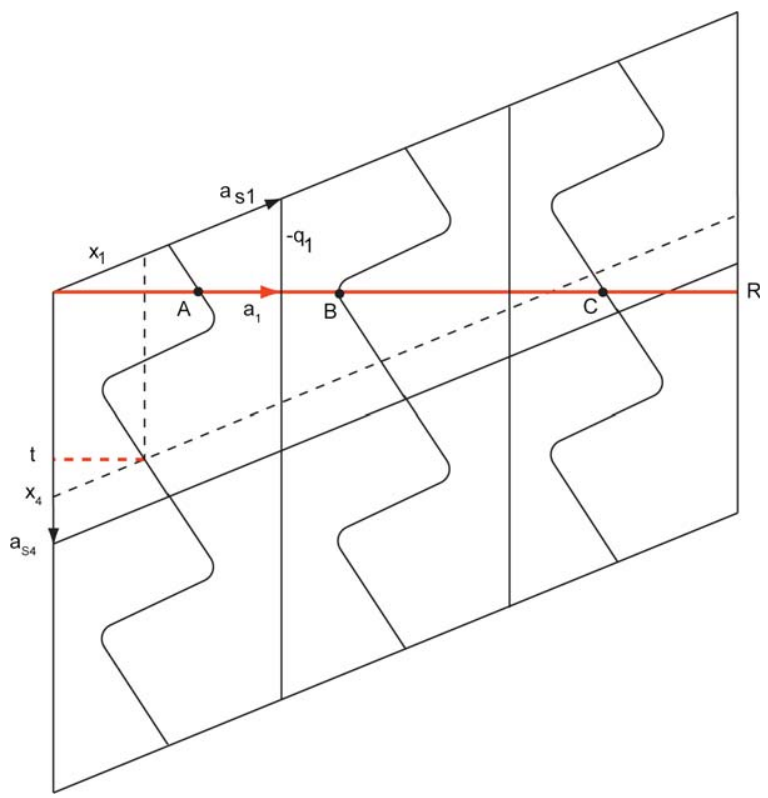

Fig. 2. Four-dimensional extension of a crystal in direct space. 
As in $3 \mathrm{~d}$, the description of a crystalline structure in superspace is based on the structure factor $F_{\mathbf{H}}$ corresponding to the Fourier transform of the electronic density of the unit cell. The expression for the structure factor is given by

$$
F_{\mathbf{H}}=\sum_{j} f_{\mathbf{H}}^{j} \exp \left(2 \pi i \mathbf{H} \cdot \mathbf{r}^{j}\right) \int_{0}^{1} \mathrm{~d} t p^{j}(t) \exp \left\{2 \pi i\left(\mathbf{H} \cdot \mathbf{u}^{j}(t)+h_{4} t\right)\right\}
$$

where the summation is over all the $j$ atoms in the unit cell with atomic scattering factor $f_{\mathrm{H}}^{j}$. The modulation $\mathbf{u}^{j}$ describes the individual atomic displacements relative to its basic position $\mathbf{r}^{j}$. The integration variable $t$ is related to the fourth dimension by the expression $x_{4}=\mathbf{q r}+t$, as seen in Fig. 2. The modulation function can also be affected by a population parameter $p^{j}$. The first part of this expression before the integral corresponds to the structure factor of a conventional crystal. The second part is specific for aperiodic structures. It contains the characteristics of the modulation function in superspace.

In the present context, we shall not deal further with the symmetry of superspace, which is also central for the description of aperiodic structures. We shall only mention that the superspace groups for 4 and 5 dimensions have been derived and tabulated [5]. Just as a (3d) periodic structure is characterised by its space group symmetry, each modulated structure is also characterised by its superspace group symmetry.

As mentioned before, the structure of $\gamma-\mathrm{Na}_{2} \mathrm{CO}_{3}$ is at the origin of the superspace concept applied to the description of aperiodic crystals. Without entering into the details of the structure solution (see e.g. Ref. [6]), we shall focus on the incommensurately modulated phase $\gamma$, which is stable over a large temperature range, from 170 to $628 \mathrm{~K}$. Below and above this range, $\delta$ - and $\beta-\mathrm{Na}_{2} \mathrm{CO}_{3}$ are periodic.

In order to get some insight into the modulated structure, let us first describe the general features of the atomic architecture. In this structure, we can distinguish three types of $\mathrm{Na}$ atoms, $\mathrm{Na} 1, \mathrm{Na} 2$ and $\mathrm{Na} 3$ (Fig. 3). $\mathrm{Na} 1$ and $\mathrm{Na} 2$ are located at
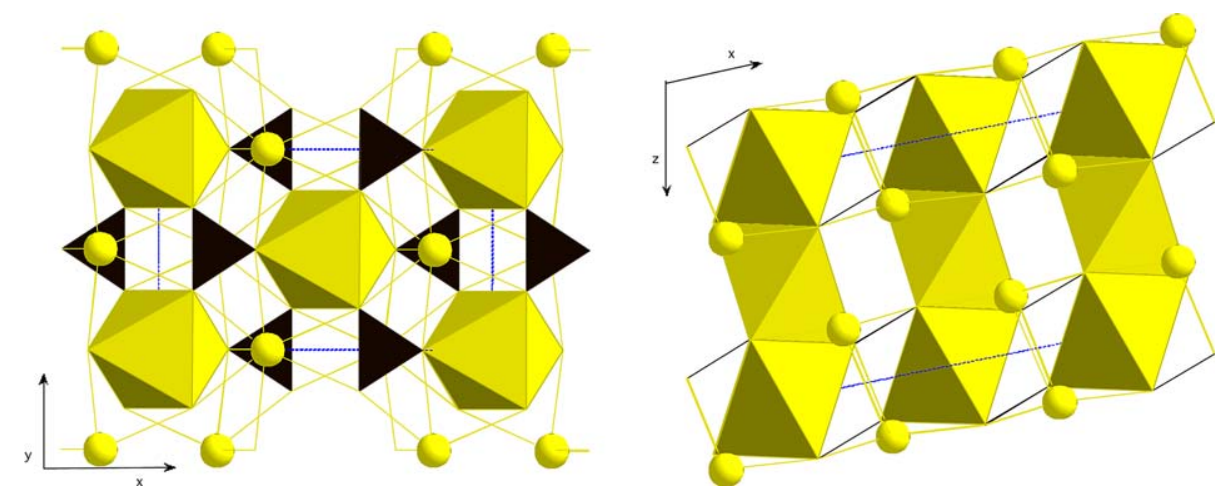

Fig. 3. $x y$ and $z x$ projections of $\mathrm{Na}_{2} \mathrm{CO}_{3}$. Na1 and $\mathrm{Na} 2$ occupy the centres of the faces sharing oxygen octahedra. The $\mathrm{CO}_{3}$ groups link the isolated columns. Na3 is represented by isolated spheres. 


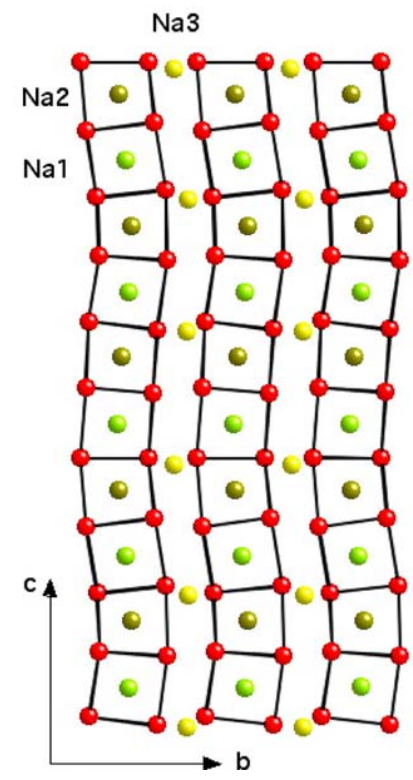

Fig. 4. Projection of the modulated structure along a. The lines linking the $\mathrm{O}$ atoms do not represent bonds but indicate the nature of the modulation. $\mathrm{C}$ atoms are omitted.

the centres of faces sharing oxygen octahedra which form isolated columns along $z$. In a column, each octahedron is alternately occupied by $\mathrm{Na} 1$ and $\mathrm{Na} 2$. The columns are linked together by $\mathrm{CO}_{3}$ groups maintaining the columns at equal distance. Contrary to Na1 and Na2, Na3 is not octahedrally coordinated but is rather surrounded by $12 \mathrm{O}$ atoms at various distances, thus giving many alternatives to form coordination polyhedra satisfying the valence requirements. This characteristic is specifically at the origin of the modulated nature of $\gamma-\mathrm{Na}_{2} \mathrm{CO}_{3}$. The new resolution of this structure [6] in the anharmonic approximation shows specifically that the modulation originates from the spatial variation of the $\mathrm{Na3}-\mathrm{O}$ coordination. The structure attempts to sequentially adopt many possible and favourable $\mathrm{Na} 3$ coordinations giving rise to the modulation not only of $\mathrm{Na} 3$ but the rest of the structure and in particular the $\mathrm{Na} 1$ and $\mathrm{Na} 2$ columns (Fig. 4). The periodicity of the modulation is temperature dependent and thus independent of the lattice constants. The origin of the aperiodic character of this structure lies specifically in the discrepancy between the four independent lattice periodicities.

\section{Back to calaverite}

In the GPP paper [1], a gnomonic projection of all the faces observed in their vast collection of specimens was also included. Gnomonic projections have the interesting property of revealing the undistorted reciprocal lattice of the corre- 
sponding crystal structure provided a suitable orientation of the specimen is available. Fig. 5 is based on table 1 in Ref. [1], which in the original version contained only the array of spots, some characterised by letters, and the vertical and horizontal lines. Apparently, the authors recognized the existence of main spots (letters in Fig. 5) forming a two dimensional reciprocal lattice. The other spots remained part of the unsolved puzzle.

After a few decades of experience in the field of aperiodic crystals, it is straightforward to complete the gnomonic projection by observing that all the spots are located on a series of oblique and parallel lines. Each oblique line can be associated with one of the main spots, and moreover, all the spots are located on positive and negative integer multiples of the vector $\boldsymbol{q}$, which is given on the figure. In other words, the complete reciprocal lattice of the aperiodic crystal can be directly observed on the gnomonic projection! It is straightforward to index each spot with

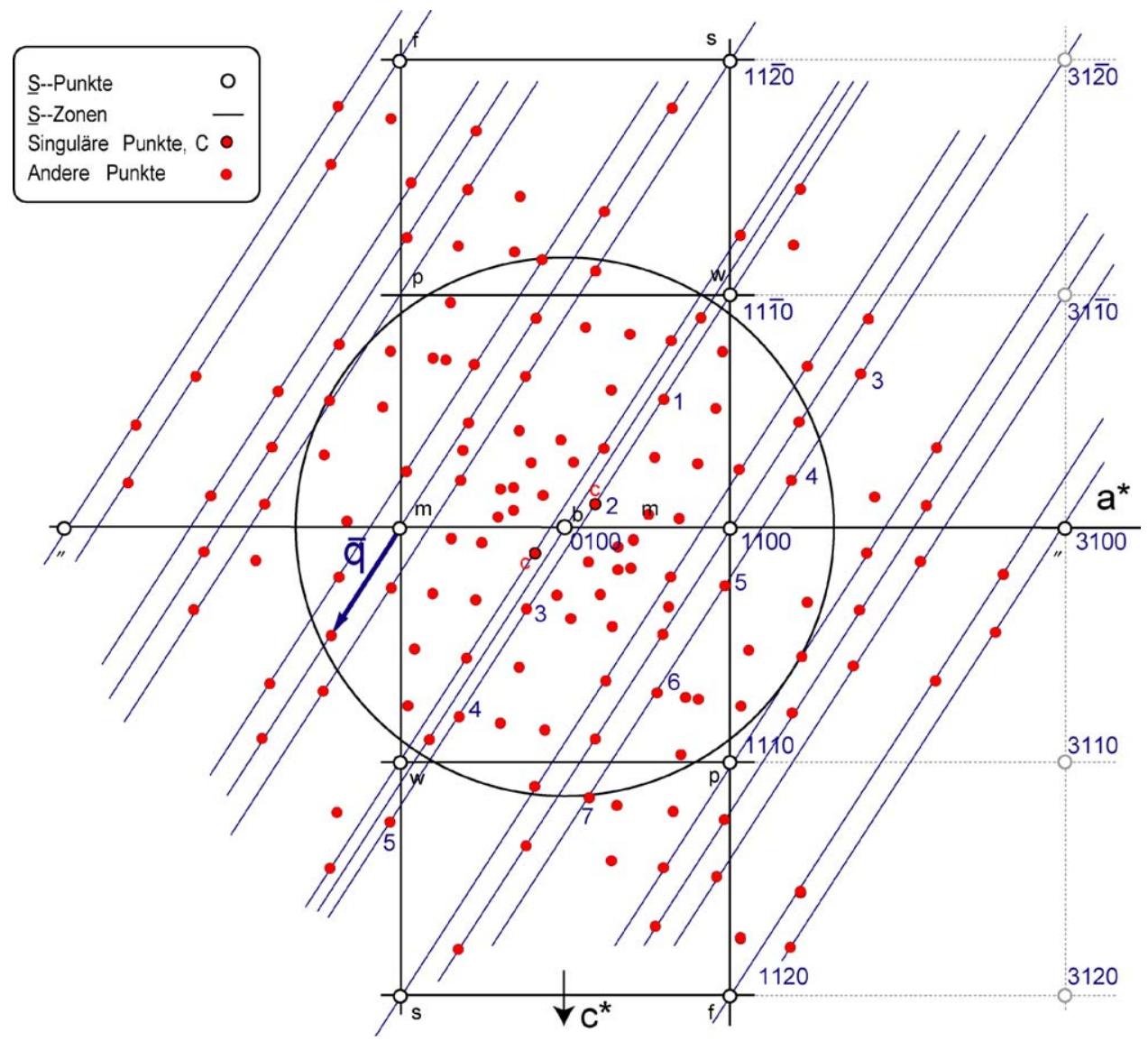

Fig. 5. Gnomonic projection of calaverite drawn according to table 1 in Ref. [1]. The oblique lines, the modulation vector $\mathbf{q}$, the integer indices and the reciprocal vectors have been added. 

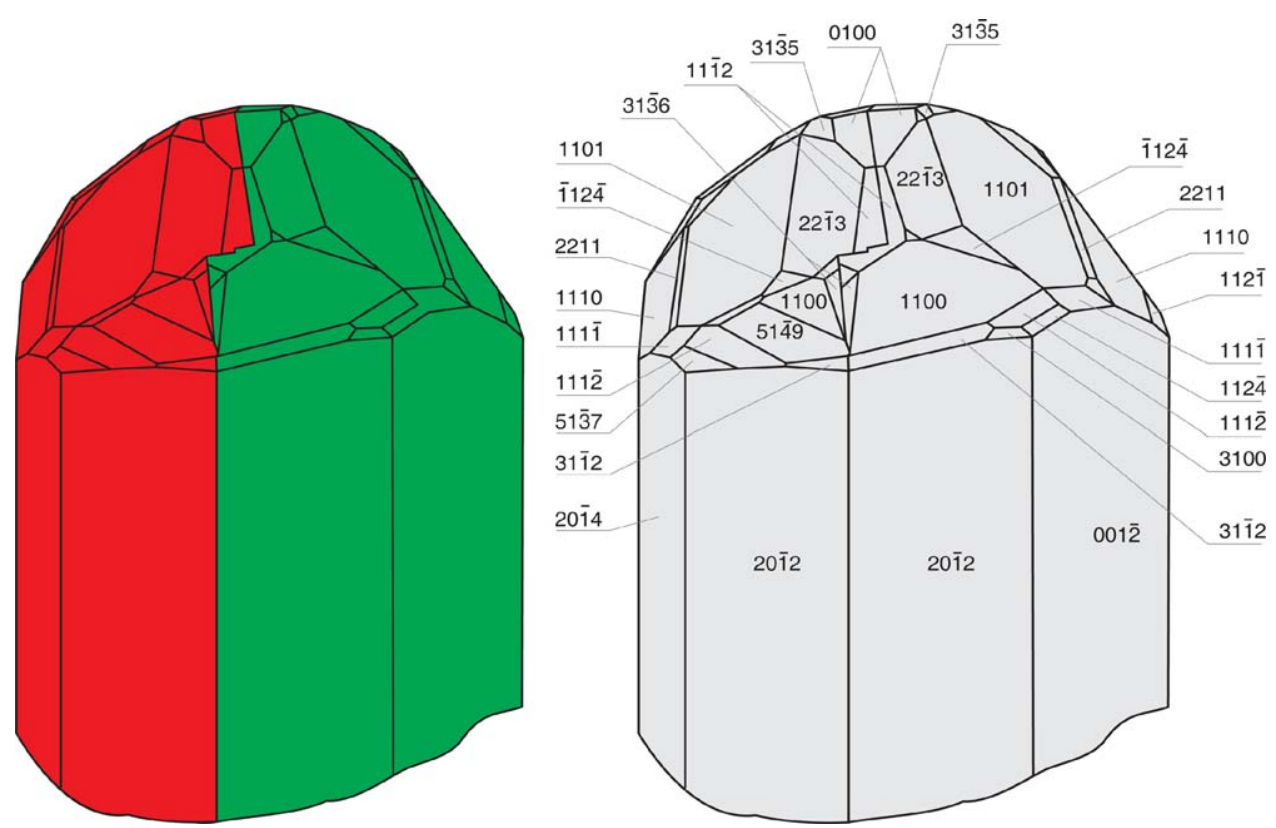

Fig. 6. The morphology of calaverite according to GPP (left) and as indexed by Janner and Dam (right). Both diagrams have been redrawn according to the original publications $[1,7]$.

four integer indices, which are included on the figure for the main reflections. The complete indexing of the crystal faces was published in 1989 by Janner and Dam [7] using four integers indices (Fig. 6). The precision of the data given by GPP also allowed Janner and Dam to estimate the correct coefficients of the modulation vector component $\boldsymbol{q}$ of calaverite as determined by X-ray diffraction [8] by Schutte and de Boer. These authors presented a complete description of the incommensurately modulated structure of calaverite on the basis of superspace formulation. They also presented very convincing arguments on the origin of the modulation in the structure by showing that the driving force of the modulation is due to valence fluctuation of the $\mathrm{Au}$ atom. Two types of $\mathrm{Au}$ atoms can be identified according to the coordination of $\mathrm{Te}, \mathrm{Au}^{\mathrm{I}}$ and $\mathrm{Au}^{\mathrm{III}}$. $\mathrm{Au}^{\mathrm{I}}$ exhibits linear coordination whereas $\mathrm{Au}^{\mathrm{III}}$ exhibits square planar coordination. The modulation is due specifically to the alternating configurations which are present in the crystal with a direction and periodicity independent of the three lattice periodicities.

\section{Concluding remarks}

The aperiodic character of calaverite and $\gamma-\mathrm{Na}_{2} \mathrm{CO}_{3}$ is by no mean exceptional in the world of condensed phases. In the last few decades, many examples of aperi- 
odic crystals have been observed, measured and fully characterised by diffraction and other methods. These structures occur in all types of crystalline material, from minerals to organic material and from metals to alloys. Their range of stability varies with pressure and temperature. They may be stable over a fraction of a degree to a few hundreds of degrees. Historically, three types of aperiodic crystals were essentially distinguished: incommensurately modulated crystals, composites and quasicrystals. It appears, however, that the distinction between these categories is getting more diffuse. Examples of crystalline structures have been described either as composite or as modulated structures.

At this point, it is important to stress that these structures should not be considered as disordered, as is often found in the literature. The discrete distribution of the diffraction spots is direct proof of the long range ordering of the structural units in the crystal. This long range is, however, not based on (3d) lattice periodicity but is due to a different nature of order. The seminal work of de Wolff, who introduced the concept of superspace to describe the structure of aperiodic crystals, was the catalyst for the new development of generalised crystallography. This field is still evolving and will probably serve us some additional surprises in the future.

Coming back to the initial claim of GPP stating that the law of rational indices is not of general validity, what can we conclude on the basis of the present knowledge? The validity of the law is still complete provided that the number of required indices corresponds to the rank of the structure and not to the space dimension. With this additional condition, each face of any aperiodic crystal can still be fully characterised by integer indices.

\section{Acknowledgements}

The help of Ivan Orlov in the preparation of the figures is gratefully acknowledged.

\section{References}

[1] V. Goldschmidt, C. Palache, M. Peacock, N. Jahrbuch, f. Mineralogie 63 (A) (1931) 1-58.

[2] G. Tunell, C.J. Ksanda, J. Wash, Acad. Sci. 26 (1936) 509-528.

[3] E. Brouns, J.W. Visser, P.M. de Wolff, Acta Cryst. 17 (1964) 614.

[4] W. van Aalst, J. den Holander, W.J.A.M. Peterse, P.M. de Wolff, Acta Cryst. Section B 32 (1976) $47-58$.

[5] International Tables of Crystallography, vol. C, second ed., Kluwer Academic Publishers, 1999.

[6] M. Dusek, G. Chapuis, M. Meyer, V. Petricek, Acta Cryst. Section B 59 (2003) 337-352.

[7] A. Janner, B. Dam, Acta Cryst. Section A 45 (1989) 115-123.

[8] W.J. Schutte, J.L. de Boer, Acta Cryst. Section B-Struct. Sci. 44 (1988) 486-494. 